\title{
Cytokine Assays
}

\author{
Theresa L. Whiteside \\ University of Pittsburgh Cancer Institute and Departments of Pathology, Immunology and Otolaryngology, \\ University of Pittsburgh School of Medicine, Pittsburgh, PA, USA
}

BioTechniques 33:S4-S15 (October 2002)

\section{INTRODUCTION}

Cytokines are low-molecular-weight glycoproteins produced by immune as well as non-immune cells. Cytokines have been shown to regulate immunologic responses, hematopoietic development, and cell-to-cell communication as well as host responses to infectious agents and inflammatory stimuli $(2,23,63,74)$. $\mathrm{Cy}$ tokines mediate interactions between various cells, and their dysregulated production might contribute to the disease pathogenesis. Under normal circumstances, cytokines are not detectable (or at low levels) in body fluids or tissues. Therefore, their presence at elevated levels of expression indicates activation of cytokine pathways associated with inflammation or disease progression.

Cytokines associated with acute diseases are distinct from those detectable in chronic conditions. Certain cytokines may be present early, others late in the disease. Consistent association of elevated cytokine levels with a disease process suggests that anti-cytokine therapy might be effective. Thus, an assessment of cytokines in body fluids, tissues, or cells of patients with various diseases is important, particularly if cytokines themselves are utilized as therapeutic agents. Today, a wide range of cytokine assays is available, providing an opportunity to evaluate the biology of cytokines and to establish their therapeutic potential.

The biology of cytokines is complex and much remains to be learned. All cytokines are pleiotropic, i.e., they have the ability to interact with a variety of cellular targets, usually via the specific receptors expressed on the surface of a target cell. Binding of cytokines to cell surface receptors initiates signaling and new RNA and protein synthesis. Cytokines are also redundant, in that the same biologic function can be executed by several distinct cytokines. This overlapping of cytokine activities, where a single cell target shows seemingly identical responses to multiple cytokines, has been referred to as "cytokine cross talk."

Cytokines are known to function as networks or cascades of interacting cytokines, which are able to induce each other. They regulate their own production via autocrine, juxtacrine, or paracrine pathways in response to microenvironmental stimuli. Cytokines induce biologic effects at minute pharmacologic doses and, once released by the producing cell, have a short half-life.
They are powerful physiologic mediators with a potential for inducing tissue damage and are local mediators meant to exert biologic effects in their microenvironment. The systemic effects of cytokines are often quite different and more dramatic than those mediated locally (25).

A division of labor exists among cytokines, in that the Th1type cytokines (e.g., IL-2, IFN- $\gamma$, IL-12, TNF- $\alpha$ ) are considered to be responsible for elimination of intracellular infections, notably those caused by viruses or parasites, modulation of organspecific autoimmune diseases or mediating allograft rejection, and recurrent abortions $(84,85)$. In contrast, Th-2-type cytokines (e.g., IL-3, IL-4, IL-5, IL-13) are involved in promoting antibody-mediated responses. A third category of cytokines, Th3, includes regulatory mediators, such as IL-10 or TGF- $\beta$, which are responsible for maintaining a balance in the host microenvironment (66). This division of labor is a reflection of cytokine polarization and appears to be useful in the classification of certain human diseases into the Th1 or Th2 categories. It also forms a basis for a concept of the "cytokine profile."

Since cytokine polarization occurs in many pathologic conditions, profiling of several cytokines might be clinically more useful than measuring one cytokine. An improved understanding of cytokine interactions has led to a consensus that a simultaneous assessment of many cytokines in a single biologic sample is desirable. Therefore, cytokine assays that best lend themselves to multiplex-type formats are slowly replacing the traditional biological and immunologic cytokine assays of the past.

\section{TYPES OF CYTOKINE ASSAYS}

Levels of cytokines in body fluids or tissues can be measured by a broad variety of assays (Table 1 ). In vivo cytokine assessment, which measures their biologic activities, is considered to be more relevant than ex vivo assays. As exemplified by the pyrogen assay for IL-1(21), in vivo assays involve injections of a cytokine into experimental animals or humans and monitoring its biologic effects. In humans, this is only possible in clinical trials utilizing cytokines for therapy. In this context, the cytokine 
Table 1. Methods Available for Assessment of Cytokines in Body Fluids, Cells, or Tissues

1. Cytokines in body fluids or cellular supernatants:

a) Bioassays and immunoassays for cytokine levels

b) Bioassays and immunoassays for cytokine soluble receptor levels

c) Definition of cytokine/cytokine receptor profiles

2. Cytokine production by populations of cells:

a) spontaneous cytokine production

b) stimulated cytokine production

c) multiparameter flow cytometry for cytokine positive cells

d) magnetic beads-based quantification or isolation of cells secreting cytokines

e) transcripts for cytokines or cytokine receptors: in situ hybridization (ISH) real time quanitative RT-PCR Northern blots RNase protection assays (RPA)

3. Single-cell assays for cytokine expression or secretion:

a) Intracytoplasmic cytokine staining (ICC)

b) ELISPOT

c) mRNA-based assays (see above)

4. Detection of cytokines in tissues:

a) Immunocytochemistry

b) Immunofluorescence

c) mRNA-based assays (see above)

5. Cytokine profiling:

a) proteomics

b) genomics

pharmacokinetics and its toxicity are often measured in lieu of its biologic activities. In general, these assays are expensive, utilize large numbers of animals, and require special facilities as well as expertise. For these reasons, they have been largely replaced by in vitro-based bioassays, which depend on the utilization of cytokine-responsive cell lines for determining biologic ef fects of body fluids obtained from animals or humans treated with cytokines (reviewed in Reference 105).

Ex vivo assays used for assessment of cytokine levels in body fluids or cellular supernatants fall into two general categories: bioassays and immunoassays. Immunoassays measure cytokine levels but not cytokine activity. Thus, although more widely used than bioassays, they are less informative. Cytokine production or cellular expression can be measured at the protein or mRNA level in cells derived from body fluids or tissues or directly in tissue biopsies. The protein-based assays are generally more informative, because mRNAs for cytokines tend to be rapidly processed and may not be detectable even with very sensitive procedures. Furthermore, assays based on cytokine production are preferable to those relying on expression not only because function is more convincing than appearance, but also because translational defects in certain pathologic conditions might result in a cytokine accumulation in the Golgi without its con- comitant secretion. Single-cell cytokine assays have gained wide acceptance, as they allow for the frequency analysis of cytokineproducing cells in populations of hematopoietic or tissue cells and for a precise identification of the cell type involved.

As mentioned above, measurements of individual cytokines are being replaced today by cytokine profiling, utilizing multiplex formats. The advent of cytokine genomics and application of cytokine genes to microarrays have already changed the way cytokines are viewed and measured. The definition of a specific cytokine profile may prove to correlate better with physiologic or pathologic events or with a specific therapeutic intervention than a single cytokine assay and thus is likely to become more clinically useful.

\section{IMMUNOASSAYS FOR CYTOKINES}

These antibody-based assays are widely available in the form of familiar commercial kits. Exemplified by enzyme-linked immunosorbent assays (ELISA), immunoassays are popular because of their acceptable specificity, sensitivity, rapid turnaround time, convenience, the ease of performance, and a relatively low cost. However, it is necessary to be aware that any immunoassay (i) is strictly dependent on the quality of the capture and detection antibodies it utilizes; (ii) might detect biologically inactive cytokines and their fragments, if they are recognized by the capture antibodies used in the assay; (iii) may be negative, if the primary structure of the antibody is altered, e.g., by glycosylation or amidation, and interferes with its binding to cytokine molecules; (iv) is often influenced by "matrix effects," which refers to nonspecific binding to the assay matrix of proteins present in complex biologic fluids that may contribute to a high assay background and thus interfere with result interpretation.

The source and quality of antibodies used for capture and detection of cytokines are by far the most important factors for the success of an immunoassay. The disparity in results observed when using ELISA kits made with antibodies from different sources is likely a result of antibody characteristics, such as its ability to bind to plastic or its affinity for the cytokine. Certain antibodies can detect soluble (secreted) as well as membranebound forms of the same cytokine, while others detect only soluble cytokine. For example, IL- $1 \beta$ is secreted as a $17-\mathrm{kDa}$ fragment, and it also exists as a larger, $35-\mathrm{kDa}$, molecule (22). ELISA formats designed to measure $1-\mathrm{kDa}$ IL-1 $\beta$ were reported to underestimate the $35-\mathrm{kDa}$ membrane-associated cytokine (43). An antibody able to detect a free, soluble cytokine may not detect the same cytokine bound to its receptor or forming immune complexes in the circulation. Certain commercially available cytokine kits detect highly elevated levels of cytokines in normal human sera. It is possible that the antibodies used in these kits measure both free and bound cytokines (75). At this time, the biologic significance of such measurements is not known, and it may be important in such cases to resort to the simultaneous use of biologic and immune assays to confirm bioactivity in the sample.

ELISA-based cytokine assays are available in both direct or indirect formats. They have largely replaced radioimmunoassays (RIA) for reasons related to the use of radioactivity (usually ${ }^{125} \mathrm{I}$ ) and the costly requirement for a gamma counter. The indirect or 


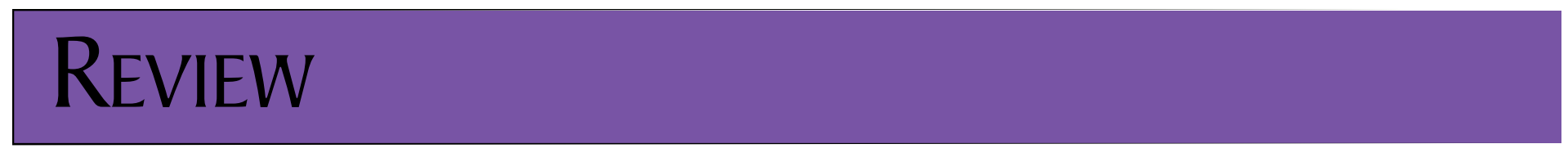

"sandwich" ELISA, in which a targeted cytokine is sandwiched between a capture antibody bound to the plate and an enzymelabeled detection antibody, is the most frequently utilized method today. The color is developed by the addition of the appropriate substrate (e.g., Reference 19).

\section{BIOASSAYS FOR CYTOKINES}

Biological activity of cytokines, regardless of whether they are available as soluble proteins, cytokine-receptor complexes, or cytokine-serum protein aggregates, is determined in cellular assays. While highly sensitive (in the picogram range), bioassays are not specific, and neutralization with cytokine-specific antibodies is necessary to confirm the identity of measured cytokines. This is because several different cytokines in the unknown sample could mediate the same biologic activity (cytokine redundancy). Bioassays are more time-consuming and labor-intensive than immunoassays. They require a tissue culture facility, because of the requirement for a target cell line, which serves as a read-out of the biologic response. Today, bioassays are mainly used for validation purposes rather than routine cytokine assessments.

Target cell lines used in cytokine bioassays are carefully selected for sensitivity to or dependence on a given cytokine. The successful performance of these assays is strictly dependent on the characteristics of cultured target cells, and any deviation from the established optimal conditions or lapse in culture sterility has detrimental effects on the assay reliability. These target cell lines may be obtained from the American Type Culture Collection (ATCC; www.atcc.org), a nonprofit organization in Manassas, VA, which serves as a repository for cell lines of various origins. The common practice of exchanging cell lines between laboratories is discouraged because of the danger of mycoplasma infection, which can alter functional characteristics and growth of cells used as targets in bioassays. It is recommended that all cell lines used as targets for cytokines are screened for mycoplasma at regular intervals, using commercially available DNA hybridization-based assays.

Bioassays may be divided into four broad categories based on the type of function measured in the target cells incubated in the presence of a cytokine: (i) assays which measure proliferation or growth inhibition; (ii) assays of cytotoxicity; (iii) assays dependent on the induction of a specific cell function, such as chemotaxis; and (iv) assays estimating the quantity of a protein induced in the target cell.

In cytokine-driven proliferation assays, growth of target cells is the endpoint: the cells stop growing unless the relevant cytokine is present. A good example is a response of B9 cells to IL-6, as described by Remick (82). These cells will not only increase in number in the presence of IL- 6 , but in the absence of IL-6, they will die out over time. Another common bioassay utilizes CTLL-2 cells for measuring the presence of IL-2 (34). Other bioassays depend on the ability of cytokines to induce death of target cells, as in a bioassay for tumor necrosis factor (44). The number of cells is measured at the end of the assay by incorporation of tritiated thymidine, manual cell counts, or by nonradioactive methods, e.g., those based on the use of MTTtetrazolium (3-[4,5-dimethylthiazol-2-yl-]2,5-diphenyltetrazolium bromide) $(65,95)$ or water-soluble tetrazolium salts, such as
XTT, MTS, or WST-1 (37,59). The results of proliferation/ growth inhibition assays are expressed in cpm (for tritiated thymidine incorporation) or in arbitrary half-maximal units (for metabolic assays) defined as the dilution that produces a halfmaximal response (81).

Certain cytokines, such as TNF- $\alpha$ or TNF- $\beta$, exert a direct cytotoxic effect on susceptible target cells $(64,80,82)$. In cytotoxicity assays, the number of target cells killed during the assay is measured and related the concentration of the cytokine in a biologic fluid. Alternatively, the uptake of a dye (e.g., naphthol blue-black or neutral red) by residual viable cells at the end of the assay can be quantified, using an ELISA reader. This response is inversely proportional to cytotoxic activity of the cytokine, and it often occurs in a log-linear fashion, where the toxicity of the cytokine declines logarithmically with its dilution. An example of this response is the WEHI 164 cell line, which is very sensitive to the toxic effects of TNF (82).

Cellular functions other than proliferation or death have also been used for measuring biologic effects of cytokines. These range from secretion of secondary cytokines (12), changes in phosphorylation of signaling molecules (88), alterations in the calcium flux (110), up-regulation of the major histocompatibility complex (MHC) molecules (32), release of cytoplasmic granules (92), induction of respiratory burst in macrophages or granulocytes (27) or promotion of chemotaxis by the chemokines $(10,48,96,97,98)$. By and large, these assays are used in research laboratories, and none has so far been adapted to a clinically applicable format.

The fourth type of a bioassay is a two-step procedure, depending on the ability of a cytokine to induce a specific cellular function, which is then measured in the next step. Antiviral assays are included in this category $(55,56)$. These involve the preincubation of susceptible cells with interferons followed by measurements of an increased survival and protection from cytopathic effects of a challenge virus, such as encephalomyocarditis virus or vesicular stomatitis virus. Target cells are human primary fibroblasts or cultured Hep 2C, WISH, or A549 cell lines, and the number of viable target cells is determined at the end of the assay. Another example is a bioassay for IL-18, which depends on the ability of IL-18 to induce IFN- $\gamma$ secretion by T lymphocytes $(24,49)$.

Bioassays for cytokines are infrequently performed, because they are demanding and labor-intensive (9). Also, the analysis of S-shaped dose-response curves generated in the bioassay requires special computer programs for analysis (87). Bioassays do not lend themselves easily to automation or a rapid throughput and are not appropriate for a large-scale screening of specimens. Nevertheless, the bioassay is a gold standard of cytokine testing. Since it measures bioactivity of cytokines, not just their presence or levels, it is the only biologically relevant cytokine assay, aside from in vivo animal-based experiments. Thus, the bioassay is most often used for confirmation and validation of results obtained in other cytokine assays.

\section{PITFALLS OF CYTOKINE ASSAYS}

A major pitfall associated with cytokine bioassays is the lack of specificity, as it may be impossible to rule out contributions of 
other cytokines present in the complex biologic mixtures even with the introduction of a neutralizing antibody (106). Target cells generally respond to a range of cytokines and growth factors, making discrimination difficult. Attempts to transfect cytokine receptor genes or gene promoters for cytokine genes induced by the measured cytokines into such target cell lines have been made to increase specificity of responses in bioassays $(1,45)$.

Another major concern of cytokine testing applicable to both immunoassays and bioassays is the presence in biologic samples of specific inhibitors of cytokines such as soluble cytokine receptors, cytokine antagonist molecules (e.g., IL-1 $\beta$ antagonist) or antibodies to cytokines $(24,28,30)$. Nonspecific inhibitors present in human sera, such as albumin, $\alpha 2$-macroglobulin, or lipids, tend to bind to cytokines, which may result in false-negative results $(7,46,60)$. A variety of procedures have been tried to remove nonspecific serum inhibitors of cytokines, including chloroform extractions (13), treatment with polyethylene glycol $(53,69)$ and simple serial dilutions of specimens. It is unclear whether the assays employing these treatments provide biologically relevant information, however. Removal of specific cytokine inhibitors by using immunoaffinity columns for soluble cytokine receptors or immune complexes may interfere with detection of cytokines and are not validated to date.

Perhaps the most common pitfalls associated with cytokine assays stem from inadequate handling of specimens. Collection and processing of samples for cytokine assays under optimal conditions is necessary, if artifacts are to be avoided $(14,99,109)$. These occur for two main reasons: cytokines are degraded or lost because of inappropriate specimen handling, or specimens are collected in a correct way but are not stored or assayed under optimal conditions. In terms of handling, the most common problem is that use of anti-coagulants such as EDTA or citrate removes divalent cations and might interfere with cytokine bioassays. However, it is best to avoid coagulation, which might activate cells or result in removal of some cytokines with the clot. Thus, plasma rather than serum should be collected and placed in the cold immediately after phlebotomy. Even if collected properly, prolonged storage in ambient temperatures, repeated freezing and thawing of samples or additions to the assays of mitogenic substances and cytostatic reagents, are all likely to interfere with cytokine testing. In ELISA, serum or plasma matrix ef fects are frequently a reason for unacceptably high backgrounds.

\section{ASSAYS FOR CYTOKINE PRODUCTION}

In vitro production of cytokines by hematopoietic cells obtained from peripheral blood or tissues may be a more biologically meaningful measure of cytokines than their plasma levels $(108,111)$. Except for situations requiring pharmacokinetic studies of exogenously administered cytokines or chronic conditions associated with continuous cytokine production, spontaneous or stimulated production of cytokines by peripheral blood mononuclear cells (PBMCs) or their subsets is a useful alternative to assays in plasma. The first is a measure of in vivo activation of these cells, while the second can be viewed as a measure of immune competence (29).

The assay for spontaneous cytokine production is based on the rationale that immune cells activated in vivo will sponta- neously produce cytokines when isolated and placed in culture. PBMCs or subsets of purified cells are incubated in medium without any additional stimulation for $24-48 \mathrm{~h}$, and the culture supernatant is then assayed for the presence of cytokines. In contrast, the assay for stimulated cytokine production is a measure of the overall ability of the tested cells to produce a cytokine. To be able to interpret the results of these assays correctly, it is necessary to establish normal ranges of cytokine production using PBMCs of normal volunteers (29). It appears that stimulated cytokine production by fresh or cryopreserved/thawed PBMCs in response to anti-CD3 $\mathrm{Ab}$ or $\mathrm{PMA} /$ ionomycin is a stable trait for healthy volunteers (86). Therefore, individuals with defects of cytokine production in response to ex vivo stimulation under uniformly controlled conditions can be identified using this approach. These assays are able to measure an overall potential of cellular populations to respond to a particular simulating agent. As more than one cytokine can be concomitantly measured in the supernatants, a cellular cytokine profile can be established as well as the kinetics profile for a cytokine production (36).

Cytokine detection in single cells is possible because of recent advances made in flow cytometry. Cell permeabilization is necessary for detection of intracytoplasmic, as opposed to cell-surfaceassociated, cytokines. A variety of such methods have been described (51), and they all aim at a relatively gentle alteration of the cell membrane to allow the antibody to reach the cytokine inside the cell. Multiparameter flow cytometry allows for detection of more than one cytokine in the same cell or for the precise def inition of the lineage of cytokine producing cells by using antibodies to surface markers. Successful staining for intracellular cytokines requires the use of $(i)$ a protein transport inhibitor, such as brefeldinA or monensin; (ii) the appropriately gentle fixation and permeabilzation of cells; (iii) anti-cytokine antibodies that bind to fixed cytokine proteins; and (iv) a stimulator protocol that generates suitably high frequencies of cytokine producing cells $(17,47,79)$. This method has been widely applied to determinations of the frequency of antigen-reactive, cytokine-producing $\mathrm{T}$ cells in the peripheral circulation or in in vitro stimulated populations of hematopoietic cells $(77,107)$.

Another flow cytometry-based method for cytokine secretion by single cells is now commercially available. This method can be performed using whole blood or freshly separated PBMCs (71). The cells stimulated with antigen for several hours to induce cytokine secretion by the antigen-responsive cells are incubated with a cytokine "catch" reagent, which is a conjugate of antiCD45 and anti-cytokine antibodies. It attaches to the surface of all leukocytes and, subsequently, a cytokine secreted by the antigen-stimulated cell binds to the catch reagent. The cytokine-producing cells are visualized by a cytokine-specific detection antibody labeled with a fluorochrome and are quantified in a flow cytometer (67). If the isolation of cytokine-secreting cells is desired, magnetic beads coated with anti-fluorochrome antibodies can be used in an additional step. The antigen-responding cells are positively selected in the magnetic field, using a special column (58). The cells recovered from the column are viable and can be used for flow analysis or cell culture. The sensitivity of this method for the detection of antigen-specific, cytokine-secreting $\mathrm{T}$ cells, which are rare in the population, is one in $10^{5}$ cells.

The most widely used assay for the assessment of cytokineproducing single cells is the enzyme-linked immunospot assay 


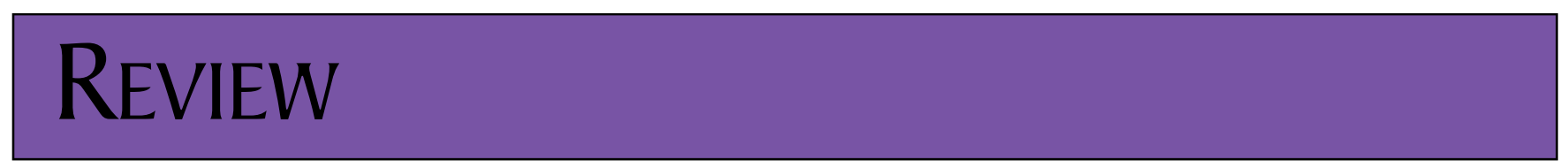

(ELISPOT), which has been developed to determine the frequency of precursor cells in fresh or cultured populations of lymphocytes capable of releasing cytokines in response to stimulation by a cognate antigen (18). ELISPOT has become an assay of choice for monitoring responses to antitumor and antiviral vaccines for several reasons. It is performed in 96-well plates and is adaptable to a high throughput, can be set up in several different formats either with unseparated PBMCs or enriched subpopulations of lymphocytes, and its results (i.e., the number of spots per well) can be acquired in an unbiased way by image analysis. It also provides an estimate of the frequency of antigen-responsive cells among those plated (40-42). The assay can detect one cytokineproducing cell per $10^{5}$ plated, and it can be performed with cryopreserved PBMCs $(4,8)$. ELISPOT assays for IFN- $\gamma$, IL-5, IL10 and other cellular products, such as granzyme B, have been useful for the assessment of cytotoxic T lymphocytes (CTLs) precursor frequencies prior and after peptide-based vaccinations in patients with cancer $(4,61,62)$. Others have used it to study the frequency of viral-specific CTLs $(26,52)$ in the peripheral circulation. In the research laboratory, this assay has almost replaced the use of more laborious cytotoxicity assays for the detection of antigen-specific $T$ cells.

The ELISPOT assay can be performed in several different formats, depending on the antigen used for vaccination and the expected frequency of antigen-specific precursor cells in the peripheral circulation. The simplest format involves adding class I MHC-restricted peptides to the HLA-matched PBMCs for the duration of a 24-h assay and reading the number of spots, which directly reflects the frequency of the peptide-responsive $T$ cells in the circulation (91). When such a frequency is low, however, PBMCs may have to be separated into $\mathrm{CD}^{+}$and $\mathrm{CD} 4^{+}$subsets, which are then tested individually in the ELISPOT assay. In vitro sensitization (IVS) of T cells with the peptide(s) of choice can be performed by culturing peptide-pulsed PBMCs over a period of one or two weeks prior to the assay. Finally, with complex antigens or antigen-antibody complexes, presentation on dendritic cells (DC) may be necessary to induce T-cell responses. Newer modifications of ELISPOT allow for simultaneous detection of several cytokines (73). The interpretation of the assay results is usually complicated by the presence of background spots formed with responder $\mathrm{T}$ cells alone, particularly following IVS in the presence of IL-2. The common practice of subtracting the number of background from experimental spots is only admissible, if the difference between the two is significant by a permutation test (93). Nevertheless, the ELISPOT assay for cytokines has been instrumental in demonstrating increases in the frequency of vaccine-responsive, and in some cases, tumor-responsive, $\mathrm{T}$ cells following vaccinations of patients with cancer (93).

\section{DETECTION OF CYTOKINES IN TISSUES}

Assays for cytokines in tissue are important, largely because the in situ cytokine profile might be more relevant to the ongoing physiologic or pathologic processes than systemic cytokine levels. Methods for in situ studies of cytokines can target proteins or mRNA, using variously labeled probes. Immunohistochemistry for cytokines is an antibody-based method $(17,90)$. Depending on the label conjugated to the antibody (i.e., an enzyme or a fluorochrome), light or fluorescent microscope is used for cytokine detection. Both direct and amplified (i.e., multilayer antibody) techniques can be selected, depending on the desired sensitivity of detection. A wide range of polyclonal or monoclonal antibodies to human cytokines are commercially available, and it is up to the user to select those that will perform optimally. Antibody selection is a crucial step, because those that perform admirably in Western blots or immunoprecipitation for cytokine proteins may not be suitable for tissue staining. Immunostaining of tissue sections or cells has to be preceded by fixation, to ensure that the cytokines are not lost during the washing procedure. However, even mild fixation might alter the conformation of a cytokine or cause its partial denaturation (100). Such altered cytokines will not be recognized by antibodies made to the cytokine in its native conformation. Therefore, antibodies selected for immunostaining must be able to recognize and bind to a cytokine after fixation. This may require prescreening of several different antibodies or even production of a new antibody.

When appropriately selected and pre-titered antibodies are used, immunostaining can provide useful information about localization of cells expressing a cytokine and the relative frequency of these cells in tissue $(102,103)$. The discrimination of patterns of cytokine expression in different tissues is not simple even under optimal experimental conditions. The stain may be discrete, e.g., confined to the Golgi zone, and it is likely to depend on the state of cellular activation, with activated cells stain ing more strongly than resting cells. Nonspecific staining has to be carefully controlled by the use of isotype control antibodies and checkerboard titrations of the primary and secondary antibodies. Furthermore, cytokines found in the cytoplasm of a cell could be internalized products of other cells, and those associated with the cell surface could be occupying cellular receptors having been produced by another cell at a distant location. Cytokines found in the Golgi zone of a cytokine-producing cell and visualized as dot-like clusters can be definitely considered as products of this cell. Often, it is useful to stain for cytokine receptors in addition to staining for the cytokine to confirm the possible presence of an autocrine loop. Finally, it is not uncommon, particularly when using paraffin-embedded specimens, to fail to detect a cytokine. Overall, immunohistochemical detection of cytokines in tissues is somewhat of an art and requires considerable experience for successful implementation.

Methods for detection of cytokine transcripts in cells or tissues generally employ reverse transcription PCR (RT-PCR), RNase protection assays (RPA), or in situ hybridization (ISH). The quality of tissue submitted for RPA, ISH, or RT-PCR remains the most critical aspect of this technology. The main disadvantage of the mRNA-based methods is the absolute requirement for a high quality of nucleic acid, which depends on special tissue/cell handling to prevent its degradation. Tissues have to be snap-frozen within minutes after surgery and never allowed to warm up for fear of activating endogenous RNases. The presence of mRNA for a cytokine in tissue is meaningful but its absence is not, as cytokine transcripts are short-lived, and their absence in the tested tissue could reflect rapid processing or degradation rather than the lack of synthesis.

Of the three methods for cytokine transcript detection, RTPCR is most commonly used, although it only identifies the presence of cytokine transcripts but not the cell containing the 


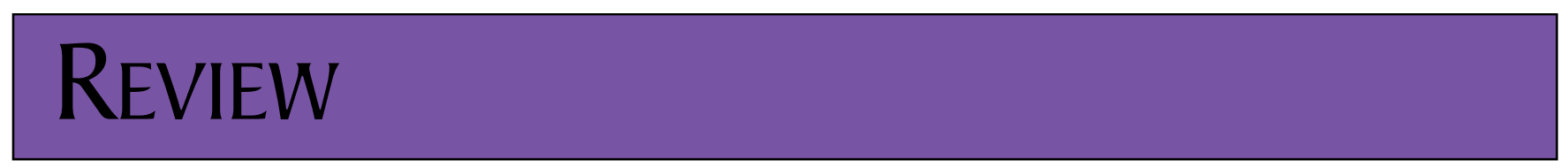

transcripts $(15,33,50,72)$. Its newer version, real-time quantitative PCR, is especially useful, because it allows for measurements of the copy number of a particular transcript relative to the expression level of a housekeeping gene or an endogenous reference, such as ribosomal RNA (rRNA) $(31,39)$. The final value obtained represents the absolute number of mRNA copies per the selected number of rRNA copies. This method enables the investigator to quantitatively compare cytokine gene expression between several different targets.

The RPA system is a highly sensitive and specific method for simultaneous detection of up to 12 different mRNA species in a single sample of total RNA $(20,89)$. The method utilizes specific riboprobes, which are generated using DNA-dependent RNA polymerases from the bacteriophages $\mathrm{T} 7$ or T3. The polymerases drive the synthesis of antisense RNA probes from DNA templates, each of distinct length and each representing a unique sequence in a specific mRNA species. The resulting linear probes are hybridized with target mRNA. After free probes and other single-stranded RNA molecules are digested with RNase, the hybridized probe/target RNA duplexes are resolved according to size on polyacrylamide gels and transferred to a nylon membrane. Quantification of the RNA fixed to the membrane by UV crosslinking follows, based on the label incorporated into antisense RNA probes (either 32P or biotin-16UTP).

ISH for cytokine transcripts is less popular than real-time PCR and perhaps more difficult to perform. Its main advantage is that it identifies cytokine transcripts within cells which produce them (54). The possibility for a combination of ISH with other methods (e.g., immunostaining) to identify or better characterize the producer cell is another advantage of this method (68). It is important to remember, however, that transcript detection is not dependent on the threshold level of cytokine production per cell, so that the presence in the same cell of cytokine mRNA transcripts and protein do not always correlate (105). Freshly harvested and quick frozen tissue biopsies are preferable over archival paraffin-embedded tissues for ISH (11). ISH can be performed as a radioactive procedure, in which the cytokinespecific oligonucleotide probes are labeled with, e.g., S35, or as a non-isotopic method with enzymatically labeled oligonucleotides $(68,102,103)$.

\section{CYTOKINE PROFILING}

Whether defined in tissues, cells, body fluids, or cellular supernatants, cytokine profiles are likely to be more informative than levels of a single cytokine. Activation of a cytokine cascade by a physiologic or pathologic stimulus usually leads to the production and release of several biologically related cytokines. Thus, IL-2, IFN- $\gamma$, TNF- $\alpha$, production characterizes Th1-type immune responses, while IL-4, IL-5, IL-10 are associated with Th2-type response $(84,85)$. An inflammatory cascade induces a release of several pro-inflammatory cytokines (21). Simultaneous measurements of several different cytokines calls for a multiplex assay format. A multiplex bead immunoassay designed to work in conjunction with the Luminex instrument $(16,101)$ utilizes distinct fluorescently labeled microspheres, each covalently linked to a cytokine-specific antibody. The combination of different beads in one tube allows for a simultaneous assess- ment of various cytokines. In the sandwich-type immunoassay, similar in principle to ELISA, the capture antibody is immobilized on microbeads. It binds the cytokine present in the sample. Biotin-conjugated detection antibody specific for another epitope on the same cytokine is then added and is followed by fluorescein-labeled streptavidin. Fluorescence bound to beads is quantified in the Luminex instrument, a flow analysis system designed to perform simultaneous multi analyte detection. The intensity of measured fluorescence is directly proportional to the concentration of the cytokine present in the specimen. The currently available panels of microbeads allow for measuring a dozen or more of selected cytokines in a single $50 \mu \mathrm{L}$ sample $(16,101)$. All of the assay components (microbeads with immobilized capture antibody, detection antibody, recombinant cytokine standards, serum diluent, streptavidin-FITC, buffers and 96-well microtiter filter plate) are included in the assay kits available from an increasing number of vendors. Customized multiplex bead assay kits are commercially available as panels (e.g., inflammatory, Th1/Th2) or in the form allowing for mixing pairs of capture and detection antibodies for detection of desired cytokines in a single specimen. The multiplex cytokine assays offer flexibility, labor/time saving and high throughput, with sensitivity and specificity that appear to be comparable to those of immunoassays for individual cytokines.

Microplate-based assays that measure cytokine-specific mRNA are also available $(35,104)$. These assays quantitate mRNA in cell lysates by using gene-specific biotin-labeled capture probes and digoxigenin-conjugated detection probes. The cytokine RNA/probe hybrid is captured on a streptavidin-coated microplate and following washing and the addition of the substrate (alkaline phosphatase conjugated to anti-digoxigenin antibody), the color is developed and read in a spectrophotometer. This method does not require mRNA purification, utilizes whole cells, is said to be more accurate than a northern blot, and has added advantages of being rapid as well as adaptable to a high sample throughput.

\section{GENOMICS AND PROTEOMICS FOR CYTOKINES}

The widening use of DNA chips or microarrays allows for the simultaneous analysis of many thousands of genes from various tissues or cells (78). This methodology can be applied to screening for expression of cytokine genes in normal or pathologic tissues or cells. An emerging technology features gene arrays to identify differentially expressed mRNA levels by comparing expression profiles of cytokines in different samples. This method can be used for the determination of cytokine genes upregulated in response to physiologic or pathologic conditions, external signals, or cellular stress. For example, using a side-byside hybridization, it is possible to simultaneously determine the profile of cytokine expression during activation, proliferation or differentiation of various cell types. The technology involves mRNA extraction from the cells or tissues and its subsequent labeling and hybridization to the microarray. The hybridized products are visualized in a phosphorimager and analyzed using software capable of comparing control with test samples and of detecting differences in expression of cytokine genes in these samples. In this way, quantitative information on the level of 


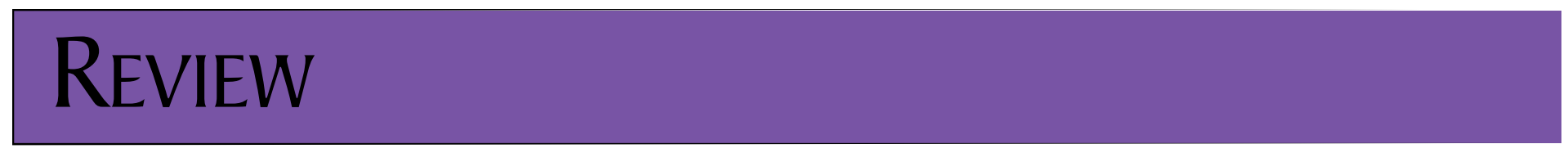

gene expression, i.e., its induction or down-regulation, is obtained. Using rapid screening of multiple samples for cytokine gene expression is likely to advance our understanding of the cytokine profiles associated with various human diseases or responses to therapies. In the experimental settings, cytokine microarrays should help in understanding changes in cytokine cascades in specific tissues or cell types responding to external signals. Although largely used as a research tool at this time, cytokine and chemokine gene arrays, applicable to screening of large numbers of biologic samples in a relatively cost-effective way, appear to be the technology of the future.

Similar to gene arrays, new technological advances combining 2-D polyacrylamide gel electrophoresis with mass spectroscopy allow for simultaneous analysis of many hundreds of proteins in body fluids or tissues (3). This approach is based on the concept that pathologic changes in tissues or cells might be reflected in distinct patterns of cytokine proteins detectable in body fluids or cellular supernatants. Still in its infancy, particularly in respect to cytokine detection, this technology has been successfully used to map proteomic changes in sera to identify patterns characteristic of ovarian cancer (76). The potential for capturing of distinct cytokine patterns or polarization in their production and relating them to a particular biologic event is clearly there, and it has an enormous experimental appeal.

\section{QUALITY CONTROL FOR CYTOKINE ASSAYS}

At the present time, cytokine assays are not performed as routine clinical laboratory procedures. Nevertheless, as with other assays for biologic materials, it is essential that cytokine assays be correctly and uniformly standardized. At the very minimum, it is necessary to establish standard operating procedures (SOPs) for cytokine measurements and to calibrate all home-grown cytokine procedures and commercial kits or reagents against the cytokine reference standards. The Biological Resources Branch (NCI-FCRDC), the Division of Microbiology and Infectious Diseases (NIAID), and the National Institute for Biological Standards and Control (United Kingdom) make available reference reagents for human cytokines (83). These reagents can be obtained free of charge upon request. To maintain the continuity of calibration with a limited quantity of the cytokine standard, an in-house reference sample can be identified, calibrated, and frozen to be used each time the assay is performed. In addition, a QC program for cytokine assays should include the following components $(5,6,109)$ :

- Establish normal ranges for cytokine levels for your laboratory, using samples obtained from normal individuals.

- Monitor inter-assay and intra-assay variability, using cytokine-rich supernatants prepared in bulk, stored at $-80^{\circ} \mathrm{C}$ in small aliquots and included in every assay. Comparisons of different lots of such supernatants with the cytokine standard or with the previously calibrated lot are made before their acceptance as QC reagents.

- "Spike" samples with the known amount of the authentic cytokine followed by measurements of its recovery to rule out the presence of inhibitors.

- Batch serially collected samples for testing in the same assay to avoid inter-assay variability.
- Participate in an external proficiency program, even one that is informally organized by several collaborating investigators.

Results of cytokine assays should be reported in picograms or nanograms of cytokine protein per milliliter and not in arbitrary units. Collection and storage of samples for cytokines under conditions that prevent their degradation or a loss of activity are important and need to be rigorously followed.

\section{CONCLUSIONS}

With a broad array of cytokine assays available, rational selection of the "right" assay becomes a major decision. Even with the current emphasis on cytokine profiling to measure cytokine cascades, issues such as the timing of the assay, the source of the specimen or requirements for specimen processing are critical. After induction, cytokine concentrations may rapidly and differentially peak and then drop to undetectable levels. The measurements of cytokines in the blood may not be optimal, while the availability of tissue biopsies or tissue exudates is usually limited. Cytokine production assays require separation of cells, and the RT-PCR-based methods call for mRNA extraction under rigorous conditions, adding complexity. The newer assays focused on cytokine polarization and cytokine profiles provide a wealth of information that is frequently difficult to interpret and correlate to the available clinical data. While cytokine and cytokine receptor profiling may ultimately be validated for clinical use, technologies for rapid screening of multiple cytokines are just beginning to be introduced. Today, it has to be acknowledged that both the choice of a cytokine assay(s) and meaningful evaluation of assay results are difficult. No single cytokine method meets the criteria for a comprehensive, biologically relevant assessment of these soluble mediators and more than one assay might be necessary for correct data interpretation.

Nevertheless, cytokine measurements have been an important part of the process of discovery and understanding of the cytokine biology and of the role various cytokines play in health and disease. Significant progress has been made in linking certain cytokines to pathologic changes in disease. This has encouraged efforts to monitor patients' plasma and/or tissues for levels of cytokines and to try and correlate assay results with clinical endpoints. The newly emerging associations between the cytokine profiles and infectious or allergic diseases provide the best examples of how cytokine determinations contribute to a better understanding of disease pathogenesis $(38,57,85)$. These clinical observations argue for serial monitoring of cytokine levels in disease and during biotherapies and for continuing attempts to define changes in cytokine profiles that occur in disease or during therapies.

Cytokines or cytokine profiles are not yet "biomarkers" of disease or disease activity, however. Interactions of cytokines with one another, with their soluble receptors or with various antagonists could, in part, explain the lack of correlations observed between systemic levels of cytokines and clinical findings. The presence of cytokine or cytokine receptor antagonists in human sera is well documented. Viral cytokine or cytokine-receptor homologues, which bind to cellular receptors or to circulating cytokines, respectively, counteracting their biologic functions, are also present in human sera (70). Even in the absence of specific or nonspecific inhibitors, excessive consumption of a cytokine 
vs. lack of its synthesis could be responsible for negative assay results. Overall, the clinical usefulness of measuring cytokine levels in plasma remains unclear, although changes of the cytokine profile in the local microenvironment might be more meaningful, based on the principle that these changes reflect functional characteristic of the cells found at the disease site.

The more recent use of cytokines as pharmacologic agents has been facilitated by an extremely rapid process of cytokine discovery, the ability to clone their genes, and the availability of cytokine recombinant proteins. Also, cytokine gene therapies represent a novel strategy of cytokine delivery to targeted tissue sites. The ability to transfer cytokine genes to cells, induce cytokine production and thus regulate essential cellular functions (growth, differentiation, apoptosis) via high affinity receptors expressed on cytokine-responsive cells in the tissue microenvironment is clearly an advantage $(2,94)$.

It is essential to bear in mind that only when validated and carefully controlled methods for cytokine detection are used can we expect to make progress in defining the role of cytokines in human disease. A critical importance of accurate and sensitive cytokine evaluation for the success of this objective cannot be overemphasized. Application of cytokine genomics and proteomics in the near future is expected to rapidly advance the field of cytokine detection. Future measurements of cytokine levels should be accompanied by monitoring of selected cellular functions associated with the presence of a given cytokine. Hypothesis-driven cytokine monitoring might provide useful clinical information and strengthen the association between distinct cytokine profiles and disease.

\section{ACKNOWLEDGMENTS}

This work was supported in part by NIH grants PO-1 DE12321 and RO-1 DE13918.

\section{REFERENCES}

1.Abe, M., J.G. Harpel, D.N. Metz, L. Lunes, D.J. Loskutoff, and D.B. Rifkin. 1994. An assay for transforming growth-factor- $\beta$ using cells transfected with a plasminogen activator inhibitor-1 promoter luciferase construct. Anal. Biochem. 216:276-282.

2.Aggarwal, B.B. and R.K. Puri (Eds.), 1995. Human Cytokines: Their Role in Disease and Therapy. Blackwell Science, Cambridge, MA.

3.Anderson, N.L, A.D. Matheson, and S. Steiner. 2000. Proteomics: applications in basic and applied biology. Curr. Opin. Biotechnol. 11:408-412.

4.Asai, T., W.J. Storkus, and T.L. Whiteside. 2000. Evaluation of the modified ELISPOT assay for interferon- $\gamma$ production in cancer patients receiving anti-tumor vaccines. Clin. Diagn. Lab. Immunol. 7:145-154.

5.Aziz, N., P. Nishanian, and J. Fahey. 1999. Levels of cytokines and immune activation markers in plasma in human immunodeficiency virus infection: quality control procedures. Clin. Diagn. Lab. Immunol. 5:755-761.

6.Aziz, N., P. Nishanian, R. Mitsuyasu, R. Detels, and J.L. Fahey. 1998. Variables that affect assays for plasma cytokines and soluble activation markers. CDLI 6:89-95.

7.Bargetzi, M.J., M. Lantz, C.G. Smith, F.M. Torti, I. Olsson, S.P. Eisenberg, and H.F. Starnes, Jr. 1993. Interleukin 1- $\beta$ induces interleukin 1 receptor antagonist and tumor necrosis factor binding protein in humans. Cancer Res. 53:4010-4013.

8.Bennouna, J., A. Hildesheim, K. Chikamatsu, W. Gooding, W.J. Storkus, and T.L. Whiteside. 2002. Application of IL-5 ELSIPOT asssays to quantification of antigen-specific T helper responses. J. Immunol. Methods
261:145-156.

9.Bienvenu, J.A, G. Monneret, M.C. Gutowski, and N. Fabien. 1998. Cytokine assays in human sera and tissues. Toxicology 129:55-61.

10. Boyden, S. 1962. The chemotacitic effect of mixtures of antibody and antigen of polymorphonuclear leukocytes. J. Exp. Med. 115:453.

11.Bromley, L., S. McCarthy, J.E. Stickland, C.E. Lewis, and J.O'D McGee. 1997. Non-isotopic in situ detection of mRNA for interleukin 4 in archival human tissue. J. Immunol. Methods 167:47-54.

12.Callard, R.E., J.G. Sheilds, and S.H. Smith. 1987. Assays for human B cell growth and differentiation factors, p. 345-364. In M.J. Clemns, A.G. Morris, and A.J.H. Gearing (Eds.), Lymphokines and Interferons. A Practical Approach. IRL Press, Oxford.

13.Cannon, J.G., J.L. Nerad, D.D. Poutsiaka, and C.A. Dinarello. 1993. Measuring circulating cytokines. J. Appl. Physiol. 75:1897-1902.

14.Cannon, J.G., J.W.M. van der Meer, D. Kwiatkowski, S. Endres, G. Lonnemann, J.F. Burke, and C.A. Dinarello. 1988. Interleukin-1 beta in human plasma: optimization of blood collection, plasma extraction, and radioimmunoassay methods. Lymphokine Res. 7:457-467.

15.Carding, S.R., D. Lu, and K. Bottomly. 1992. A polymerase chain reaction assay for the detection and quantification of cytokine gene expression in small numbers of cells. J. Immunol. Methods 151:277-287.

16.Carson, R.T. and D.A.A.Vignali. 1999. Simultaneous quantitation of $15 \mathrm{cy}-$ tokines using a mulitplexed flow cytometric assay. J. Immunol. Methods 227:41-52.

17.Chupp, G.L., E.A. Wright, D.Wu, M. Vallen-Mashikian, W.W. Cruikshank, D.M. Center, H. Kornfeld, and J.S. Berman. 1998. Tissue and T cell distribution of precursor and mature IL-16. J. Immunol. 161:3114-3119.

18.Czerkinsky, C., G. Andersson, H.P. Ekre, L.A. Nilsson, L. Klareskog, and O. Ouchterlony. 1988. Reverse ELISPOT assay for clonal analysis of cytokine production. I. Enumeration of $\gamma$-interferon-secreting cells. J. Immunol. Methods 110:29-36.

19.DeForge, L.E. and D.G. Remick. 1991. Sandwich ELISA for detection of picogram quantities of interleukin-8. Immunol. Invest. 20:89-97.

20.Dent, A.L., A.L. Shaffer, X. Yu, D. Allman, and L.M. Staudt. 1997. Control of inflammation, cytokine expression and germinal center formation by Bcl-6. Science 276:589-592.

21.Dinarello, C.A. 1991. Interleukin-1 and interleukin-1 antagonism. Blood 72:1627-1652.

22.Dinarello, C.A. 1992. ELISA kits based on monoclonal antibodies do not measure total IL-2 synthesis. J. Immunol. Methods 148:255-259.

23.Dinarello, C.A., M.J. Kluger, and M.C. Powanda. 1990. The Physiological and Pathological Effects of Cytokines. Liss, New York.

24.Dinarello, C.A., D. Novick, A.J. Puren, G. Fantuzzi, L. Shapiro, H. Muhl, D.Y. Yoon, L.L. Reznikov, et al. 1998. Overview of interleukin-18: more than an interferon-gamma inducing factor. J. Leukocyte Biol. 63:658-664.

25.Dinarello, C.A. and S.M. Wolff. 1993. Mechanisms of disease: the role of interleukin 1 in disease. N. Engl. J. Med. 328:106-113.

26. Doherty, P.C. 1998. The numbers game for virus-specific CD8+ T cells. Science 280:227.

27.Doyle, A., M. Stein, S. Keshav, and S. Gordon. 1995. Assays for macrophage activation by cytokines, p. 269-278. In F.R. Balkwill (Ed.), Cytokines: A Practical Approach. IRL Press, Oxford.

28.Engelberts, I., S. Stephens, G.J. Francot, L.I.C. van der, and W.A. Buurman. 1991. Evidence for different effects of soluble TNF-receptors on various TNF measurements in human biological fluids [letter]. Lancet 338:515516.

29.Friberg, D., J. Bryant, W. Shannon, and T.L. Whiteside. 1994. In vitrocytokine production by normal human peripheral blood mononuclear cells to measure their immunocompetence and state of activation. Clin. Diagn. Lab. Immunol. 1:261-268.

30.Gallay, P., J.P. Mach, S. Carrel. 1991. Characterization and detection of naturally occurring antibodies against IL-1 alpha and IL-1 beta in normal human plasma. Eur. Cytokine Network 2:329-338.

31.Gibson, U.E, C.A. Heid, and P.M. Williams. 1996. A novel method for real time quantitative RT-PCR. Genome Res. 6:995-1001.

32.Gibson, U.E. and S.M. Kramer. 1989. Enzyme-linked bio-immunoassay for IFN- $\gamma$ by HLA-DR induction. J. Immunol. Methods 125:105-131.

33. Gilliand, G., S. Perrin, K. Blanchard, and H.F. Bunn. 1990. Analysis of cytokine mRNA and DNA: detection and quantitation by competitive polymerase chain reaction. Proc. Natl. Acad. Sci. USA 87:2725-2729.

34.Gillis, S., M.M. Ferm, W.E. Ou, and K.A. Smith. 1978. T cell growth factor: parameters of production and a quantitative microassay for activity. J. 


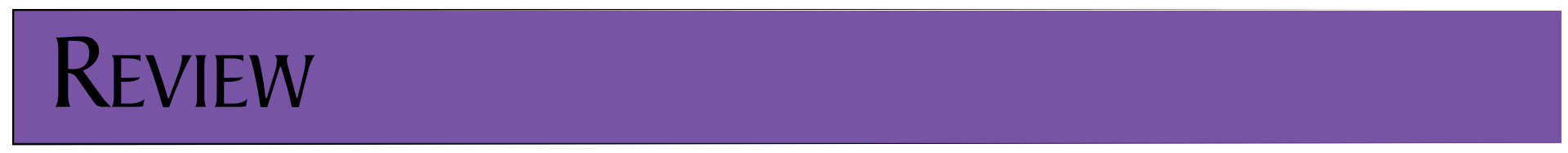

Immunol. 120:2027.

35.Gogos, C.A., E. Drosou, H.P. Bassaris, and A. Skoutelis. 2000. Pro-verus anti-inflammatory cytokine profile in patients with severe sepsis: a marker for prognosis and future therapeutic options. J. Infect. Dis. 181:176-80.

36. Gonzalez, S., L. Beck, and N. Wilson. 1994. Comparison of interferon- $\gamma$ and interleukin-4 production by peripheral blood mononuclear cells and isolated T-cells after activation with polyclonal T-cell activators. J. Clin. Lab. Anal. 8:277-283.

37.Goodwin, C.J., S.J. Holt, S. Downes, and N.J. Marshall. 1995. Microculture tetrazolium assays: a comparison between two new tetrazolium salts, XTT and MTS. J. Immunol. Methods 179.95-103.

38.Grewe, M., C.A. Bruijnzeel-Koomen, E. Schopf, T. Thepen, A.G. Langeveld-Wildschut, T. Ruzicka, and J. Krutmann. 1998. A role for TH1 and Th2 cells in the immunopatheogenesis of atopic dermatitis. Immunol. Today 19:539-361.

39.Heid, C.A., J. Stevens, K.J. Livak, and P.M. William. 1996. Real time quantitiative PCR. Genome Res. 6:986-994.

40.Herr, W., B. Linn, N. Leister, E. Wandel, K.-H. Meyer zum Buschenfelde, and T. Wolfel. 1997. The use of computer-assisted video image analysis for the quantitation of CD $8+T$ lymphocytes producing tumor necrosis factor- $\alpha$ spots in response to peptide antigens. J. Immunol. Methods 203:141-151.

41.Herr, W., J. Schneider, L.W. Ansgar, E. Wandel, K.-H. Meyer zum Buschenfelde, and T. Wolfel. 1996. Dectection and quantificaiton of bloodderived $\mathrm{CD}^{+} \mathrm{T}$ lymphocytes secreting tumor necrosis factor- $\alpha$ in response to HLA-A2.1-binding melanoma and viral peptide antigens. J. Immunol. Methods 191:131-142.

42.Herr, W., T. Wolfel, K.-H. Heike, K.-H. Meyer zum Buschenfelde, and A. Kunth. 1994. Frequency analysis of tumor-reactive cytotoxic T lymphocytes in peripheral blood of a melanoma patient vaccinated with autologous tumor cells. Cancer Immunol. Immunother. 39:93-99.

43.Herzyk, D.J., A.E. Berger, J.N. Allen, and M.D. Wewers. 1992. Sandwich ELISA formats designed to detect $17 \mathrm{kDa}$ IL-1 beta significantly underestimate $35 \mathrm{kDa}$ IL-1 beta [see comments]. J. Immunol. Methods 148:243-254.

44.Higuchi, M., S. Singh, and B.B. Aggarwal. 1995. Characterization of the apoptotic effects of human tumor necrosis factor: development of highly rapid and specific bioassay for human tumor necrosis factor and lymphotoxin using human target cells. J. Immunol. Methods 178:173-181.

45.Hu-Li, J., J. Ohara, C. Watson, W. Tsang, and W.E. Paul. 1989. Derivation of a T cell line that is highly responsive to IL-4 and IL-2 (CT.4R) and of an IL-2 hyporesponsive mutant of that line (CT.4S). J. Immunol. Methods 142.800 .

46.James, K. 1990. Interactions between cytokines and $\alpha-2$ macroglobulin. Immunol. Today 11:163-166.

47.Jung, T., U. Schauer, C. Heusser, C. Neumann, and C. Rieger. 1993. Detection of intracellular cytokines by flow cytometry. J. Immunol. Methods 159.197-207.

48.Junger, W.G., T.A. Cardoza, F.C. Liu, D.B. Hoyt, and R. Goodwin. 1993. Improved rapid photometric assay for quantitative measurement of PMN migration. J. Immunol. Methods 160:73.

49.Konishi, K., F. Tanabe, M. Taniguchi, H. Yamauchi, T. Tanimoto, M. Ikeda, K. Orita, and M. Kurimoto. 1997. A simple and sensitive bioassay for the detection of human interleukin-18/interferon-gamma-inducing factor using human myelomonocytic KG-1 cells. J. Immunol. Methods 209:187191

50.Kruse, N., M. Pette, K. Toyka, and P. Rieckman. 1997. Quanitfication of cytokine mRNA expression by RT-PCR in samples of previously frozen blood. J. Immunol. Methods 210:195-203.

51.Labalette-Houache, M., G. Torpier, A. Capron, and J.P. Dessaint. 1991. Improved permeabilisation procedure for flow cytometric detection of internal antigens. Analysis of interleukin-2 production. J. Immunol. Methods 138:143-53.

52.Lalvani, A., R. Brookes, S. Hambleton, W.J. Britton, A.V. Hill, and A.J. McMichael. 1997. Rapid effector function in CD8+ memory T cells. J. Exp. Med. 186:859-864.

53.Larrick, J.W. 1989. Native interleukin 1 inhibitors. Immunol. Today 10:6166.

54.Lewis, C.E., and A. Campbell. 1995. Visualising the production of cytokines and their receptors by single human cells, p. 339-356. In F.R. Balkwill (Ed.), Cytokines: A Practical Approach. IRL Press, Oxford.

55.Lewis, J.A. 1995. Antiviral activity of cytokines, p. 129-141. In F.R. Balkwill (Ed.), Cytokines. A Practical Approach. IRL Press, Oxford.

56.Lewis, J.A. 1995. A sensitive biological assay for interferons. J. Immunol.
Methods. 185:9-17.

57.Lucey, D R., M. Clerici, and G.M. Shearer. 1996. Type 1 and type 2 cytokine dysregulation in human infections, neoplastic and inflammatory dis eases. Clin. Microbiol. Rev. 9:532-562.

58.Manz, R., M.Assenmadrer, E. Pfluger, S. Miltenyis, and A. Radbrichi. 1995. Analysis and sorting of live cells according to to secreted molecules relocated to a cell-surface affinity matrix. Proc. Natl. Acad. Sci. USA 92:19211925.

59.Marshall, N.J., C.J. Goodwin, and S.J. Holt. 1995. A critical assessment of the use of microculture tetrazolium assays to measure cell growth and function. Growth Regul. 5:69-84.

60.May, L.T., H. Viguet, J.S. Kenney, N. Ida, A.C. Allison, and P.B. Sehgal. 1992. High levels of "complexed" interleukin 6 in human blood. J. Biol Chem. 267:19698-19704.

61.Meidenbauer, N., D.T. Harris, L.E. Spitler, and T.L. Whiteside. 2000. Generation of PSA-reactive effector cells after vaccination with a PSA-based vaccine in patients with prostate cancer. Prostate 43:88-100.

62.Meidenbauer, N, W. Gooding, L.E. Spitler, D. Harris, and T.L. Whiteside. 2002. Recovery of $\zeta$ chain expression and changes in spontaneous IL-10 produciton after PSA-based vaccines in patients with prostate cancer. Br. J. Cancer $86: 168-178$.

63. Meager, T. 1998. The Molecule Biology of Cytokines. John Wiley \& Sons, New York

64.Mire-Sluis, A.R. and R. Thorpe. 1998. Laboratory protocols for the quantitation of cytokines by bioassay using cytokine responsive cell lines. J. Immunol. Methods 211:199-210.

65.Mosmann, T. 1983. Rapid colorimetric assay for cellular growth and survival: application to proliferation and cytotoxicity assays. J. Immunol. Methods 65:55-63.

66.Mosmann, T.R., L. Li, and S. Sad. 1997. Functions of CD8 T-Cell subsets secreting different cytokine patterns. Semin. Immunol. 9:87-92.

67.Munz, C., K.L. Bickham, M. Subklewe, M.L. Tsang, A. Chahroudi, M.G. Kurilla, M. O'Donnell, and R.M. Steinman. 2000. Human CD4(+) T lymphocytes consistently respond to the latent Epstein-Barr virus nuclear antigen EBNA1. J. Exp. Med. 191:1649-1660.

68.Naylor, M.S., M. Relf, and F.R. Balkwill. 1995. Northern analysis, ribonuclease protection, and in situ analysis of cytokine messenger RNA, p. 35-56. In F.R. Balkwill (Ed.), Cytokines: A Practical Approach. IRL Press, Oxford.

69.Nemzek, J.A., D.E. Newcomb, D.R. Call, and D.G. Remick. 1999. Plasma interference in an enzyme-linked immunosorbent assay using a commercial matched antibody pair. Immunol. Invest. 28:209-221.

70.Nicholas, J., V.R. Ruvolo, W.H. Burns, G. Sandford, X. Wan, D. Ciufo, S.B. Hendrickson, H.G. Guo, et. al. 1997. Kaposi's sarcoma-associated human herpesvirus- 8 encodes homologues of macrophage inflammatory protein-1 and interleukin-6. Nat. Med. 3:287-292.

71. Oelke, M., U. Moehrle, J. Chen, D. Behringer, V. Cerundolo, A. Lindemann, and A. Mackensen. 2000. Generation and purification of CD8 ${ }^{+}$ melan-A-specific cytotoxic T lymphocytes for adoptive transfer in tumor immunotherapy. Clin. Cancer Res. 5:1997-2005.

72.O'Garra, A., and P. Vierra. 1992. Polymerase chain reaction for detection of cytokine gene expression. Curr. Opin. Immunol. 4:211-215.

73. Okamoto, Y., T. Abe, T. Niwa, S. Mizuhashi, and M. Nishida. 1998. Development of a dual color enzyme-linked immunospot assay for simultaneous detection of murine $\mathrm{T}$ helper type 1 - and $\mathrm{T}$ helper type 2-cells. Immunopharmacology 39:107-116.

74.Oppenheim, J.J. 1993. In J.J. Oppenheim, J.L. Rossio, and A.J.H. Gearing (Eds.), Clinical Applications of Cytokines. Role in Pathogenesis, Diagnosis and Therapy. Oxford University Press. New York, N.Y.

75.Paciotti, G.F., D. Baron, J. Licinio, L. Tamarkin, M.L. Wong, P.W. Gold, M.E. Altemus, and D. Rubinwo. 1992. Novel enzyme immunoassays for the detection of the cytokine sinterleukn $1-\alpha$ and interleukin 2 in the circulation of normal subjects: 24-hour profiles. Prog. Neuroendocrine Immunol. 5:21-30.

76.Petricoin, E.F.III, A.M. Ardekani, B.A. Hitt, P.J. Levine, V.A. Fusaro, S.M. Steinberg, G.B. Mills, C. Simone, et. al. 2002. Use of proteomic patterns in serum to identify ovarian cancer. Lancet 359:572-577.

77.Picker, L.J., M.K. Singh, Z. Zdraveski, J.R. Treer, S.L. Waldrop, P.R. Bergstresser, and V.C. Maino. 1995. Direct demonstration of cytokine synthesis heterogeneity among human memory/effector T cells by flow cytometry. Blood 86:1408-1419.

78.Pollack, J.R., C.M. Perou, A.A. Alizadeh, M.B. Eisen, A. Pergamenschikov, C.F. Williams, S.S. Jeffrey, D. Botstein, et al. 1999. Genome-wide analysis 
of DNA copy-number changes using cDNA microarrays. Nat. Genet. 23:4146.

79.Prussin, C. 1997. Cytokine flow cytometry: understanding cytokine biology at the single cell level. J. Clin. Immunol. 17:195-204.

80. Randall, L.A., M. Wadhwa, R. Thurpe, and A.R. Mire-Sluis. 1993. a novel sensitive bioassay for transforming growth factor- $\beta$. J. Immunol. Methods 164:61-67.

81.Remick, D.G., W.E. Scales, S.W. Chensue, and S.L. Kunkel. 1989. The pathophysiology of interleukins and tumor necrosis factors, p. 41-89. In M. Sayeed (Ed.), Cellular Pathophysiology. CRC Press, Boca Raton, FL.

82. Remick, D.G. 2002. Protein analysis and bioassays of cytokines and cytokine receptors, p. 320-337. In R.R. Rose, R.G. Hamilton, and B. Detrick (Eds.), Manual of Clinical Laboratory Immunology, 6th edition. ASM Press, Washington, DC.

83. Reynolds, C.W. 1997. Development and use of cytokine and growth factor potency standards, p. 357-359. In N.R. Rose, E.C. deMariano, J.D. Folds, H.C. Lane, and R.M. Nakamura (Eds.), Manual of Clinical Laboratory Immunology, 5th edition. ASM Press, Washington, DC.

84. Romagnani, S. 1997. The Th1/Th2 paradigm. Immunol. Today 18:263266.

85.Romagnani, S. 1997. The Th1/Th2 Paradigm in Disease. R.G. Landes, Austin.

86. Rossi, M., P. Bonino, T. Whiteside, W. Flynn, and L. Kuller. 1993. Determination of cytokine production variability in normal individuals over time. J. Am. Geriatr. Soc. 41:SA3.

87.Rossio, J.L. 1997. Cytokines and immune cell products, p. 348-356. In N.R. Rose, et al. (Eds.), Manual of Clinical Laboratory Immunology, 5th edition. ASM Press, Washington, DC.

88.Sadick, M.D., M.X. Sliwkowski, A. Nuijens, L. Bald, N. Chaing, J.A. Lofgren, and W.L.T. Wong. 1996. Analysis of heregulin-induced ErbB2 phosphorylation with a high-throughput kinase receptor activation enzymelinked immunosorbant assay. Anal. Biochem 235:207-214.

89.Sallusto, R., D. Lenig, C.R. Mackay, and A. Lanzavecchia. 1998. Flexible programs of chemokine receptor expression on human polarized T helper 1 and 2 lymphocytes. J. Exp. Med. 187:875-883.

90.Sander, B., J. Andersson, and U. Andersson. 1991. Assessment of cytokines by immunofluorescence and the paraformaldehyde-saponin procedure. Immunol. Rev. 119:65-93.

91.Scheibenbogen, C., K.-H. Lee, S. Stevanovic, M. Witzens, M. Willhauck, V. Waldmann, H. Naeher, H.-G. Rammensee, et al. 1997. Analysis of the T-cell response to tumor and viral peptide antigens by an IFN- $\gamma$-ELISPOT assay. Int. J. Cancer 71:1-5.

92.Schroder, J.M., U. Mroweitz, E. Morita, and E. Christophers. 1987. Puriff cation and partial biochemical characterisation of a human monocyte-derived, neutrophil-activating peptide that lacks interleukin 1 activity. J.Immunol. Methods 139:3474-3483.

93.Schultes, B.C., A. Gordon, T. Ehlen, C.F. Nicodemus, H. Fingert, R. Edwards, and T.L. Whiteside. 2002. Induction of tumor-and CA125-specific $T$ cell responses in patients with epithelial ovarian cancer (EOC) treated with OvaRex ${ }^{\circledR}$ Mab-B43.13. Proceedings of the American Association for Cancer Research 43:144.

94.Suminami, Y., E.M. Elder, M.T. Lotze, and T.L. Whiteside. 1995. In situ IL4 gene expression in cancer patients treated with a genetically-modified tumor vaccine. J. Immunother. 17:238-248.

95.Tada, H., O. Shiho, K. Kuroshima, M. Koyama, and K. Tsukamoto. 1986. An improved colorimetric assay for interleukin 2. J. Immunol. Methods 93:157-165.

96.Taub, D.D., M.L. Key, D. Clark, and S.M. Turcovski-Corrales. 1995. Chemotaxis of T lymphocytes on extracellular matrix proteins. Analysis of the in vitro method to quantitate chemotaxis of human T cells. J. Immunol. Methods 184:187-198.

97.Taub, D.D., P. Proost, W.J. Murphy, M. Anver, D.L. Longo, J. VanDamme, and J.J. Oppenheim. 1995. Monocyte hemotactic protein-1 (MCP-1)-2 and -3 are hemotactic for human T lymphocytes. J. Clin. Invest. 95:1370-1376.

98. Taub, D.D., A.R. Lloyd, K.Conlon, J.M. Wang, J.R. Ortaldo, A. Harada, K. Matsushima, D.J. Kelvin, et al. 1993. Recombinant human interferoninducible protein 10 is a chemoattractant for human monocytes and T lymphocytes and promotes $\mathrm{T}$ cell adhesion to endothelial cells. J. Exp. Med. 177:1809-1814.

99.Thavasu, P.W., S. Longhurst, S.P. Joel, M.L. Slevin, and F.R. Balkwill. 1992. Measuring cytokine levels in blood: importance of anticoagulants, pro- cessing and storage conditions. J. Immunol. Methods 153:115-124.

100.Thorpe, R., M. Wadhwa, C. Bird, and A.R. Mire-Sluis. 1992. Detection and measurement of cytokines. Blood. Rev. 6:133-148.

101.Vignali, D.A.A. 2000. Multiplexed particle-based flow cytometric assay. J. Immunol. Methods 243:243-255.

102.Vitolo, D., A. Kanbour, J.T. Johnson, R.B. Herberman, and T.L. Whiteside. 1994. In situ hybridization for cytokine gene transcripts in the solid tumor microenvironment. Eur. J. Cancer 3:371-377.

103.Vitolo, D., T. Zerbe, A. Kanbour, C. Dahl, R.B. Herberman, and T.L. Whiteside. 1992. Expression of mRNA for cytokines in tumor-infiltrating mononuclear cells in ovarian adenocarcinoma and invasive breast cancer. Int. J. Cancer 51:573-580.

104.Vukmanovic-Stejic, M., B. Vyas, P. Gorak-Stolinska, A. Noble, D.M. Kemeny. 2000. Human Tc1 and Tc2/Tc0 CD8 t-cell clones display distinct cell surface and functional phenotypes. Blood 95:231-240.

105.Wadhwa, M. and R. Thorpe. 1998. Assays for cytokines, p. 855-884. In A.W. Thomson (Ed.), The Cytokine Handbook, 3rd edition. Academic Press, New York.

106.Wadhwa, M., C. Bird, L. Page, A.R. Mire-Sluis, and R. Thorpe. 1995. Quantitative biological assays for individual cytokines, p. 357-392. In F.R Balkwill (Ed.) Cytokines. A Practical Approach. IRL Press, Oxford.

107.Waldrop, S.L., C.J. Pitcher, D.M. Peterson, V.C. Maino, and L.J. Picker. 1997. Determination of antigen-specific memory/effector CD4+ T cell frequencies by flow cytometry: evidence for a novel, antigen-specific homeostatic mechanism in HIV-associated immunodeficiency. J. Clin. Invest. 99:1739-1750.

108. Whiteside, T.L. 1994. Cytokine measurements and interpretation of cytokine assays in human disease. J. Clin. Immunol 14:327-339.

109.Whiteside, T.L. 1994. Cytokines and cytokine measurements in a clinical laboratory. Clin. Diagn. Lab. Immunol. 1:257-260.

110.Yu, C.R., K.W. Peden, M.B. Zaitseva, H. Golding, and J.M. Farber. 2000. CCR9A and CCR9B: two receptors for the chemokine CCL25/TECK/Ck beta-15 that differ in their sensitivities to ligand. J. Immunol. 164:1293-305.

111.Zhang, M., M.K. Gately, E. Wang, J. Gong, S.F. Wolf, S. Lu, R.L. Modlin, and P.F. Barnes. 1994. Interleukin 12 at the site of disease in tuberculosis. J. Clin. Invest. 93:1733-1739.

\section{Address correspondence to:}

Dr. Theresa L. Whiteside

University of Pittsburgh Cancer Institute

W1041 Biomedical Science Tower

200 Lothrop Street

Pittsburgh, PA 15213-2582, USA

e-mail:whitesidetl@msx.upmc.edu 